Supporting Material to

\title{
Growth of abraded crystals tracked in three dimensions
}

Simon A. Schiele, Ricardo Hupfer, Frederik Luxenburger, Heiko Briesen*

Chair of Process Systems Engineering, Technical University of Munich, Gregor-Mendel-Str. 4, 85354, Freising, Germany

*Corresponding Author

\section{Appendix I Algorithm to calculate Distances between Meshes}

Meshed surfaces were obtained from voxel based $\mu \mathrm{CT}$ images of the crystals. These meshes contained roughly $10^{5}$ triangles. We describe in the main text that we calculated distances of triangulation elements of a smaller mesh (number of triangulation elements $N_{S}$, index i) to a larger mesh (number of triangulation elements $N_{l}$, index $\mathrm{j}$ ). The small mesh lays within the larger one. Distances were calculated by constructing lines $\boldsymbol{g}_{\boldsymbol{i}}$ for each of the $N_{s}$ triangles of the inner mesh with $1 \leq i \leq N_{s}$.

$$
\boldsymbol{g}_{i}(d)=\overline{\boldsymbol{p}}_{i}+d \cdot \boldsymbol{n}_{i}
$$

Where $\boldsymbol{g}_{i}$ are points on the lines, $\overline{\boldsymbol{p}}_{i}$ is the center point of a triangle $i$ and $\boldsymbol{n}_{i}$ is the corresponding normal vector. Planes $\boldsymbol{E}_{j}$ were constructed from the $N_{l}$ triangles of the outer mesh with $1 \leq j \leq$ $N_{l}$.

$$
\boldsymbol{E}_{j}: \quad h_{j}=\boldsymbol{e}_{j} \circ \boldsymbol{n}_{j}
$$

Where $h_{j}$ is the distance of the plane to the origin, $\boldsymbol{e}_{j}$ is any point on the plane and $\boldsymbol{n}_{j}$ the normal vector of the plane. For each pair of a plane and a line a distance $d_{i, j}$ for $\boldsymbol{E}_{j} \cap \boldsymbol{g}_{i}$ was calculated.

$$
d_{i, j}=\frac{h_{j}-\overline{\boldsymbol{p}}_{i} \circ \boldsymbol{n}_{j}}{\boldsymbol{n}_{i} \circ \boldsymbol{n}_{j}}
$$

The result of our algorithm was a vector $d_{i, j} \cdot \boldsymbol{n}_{i}$ for every $i . j$ is chosen such that the intersection point of the plane and line $\boldsymbol{g}\left(d_{i, j}\right)$ is within the triangle $j$. Latter is checked by a method $^{1}$ that can be described by setting up the plane equation for a plane $j$ as

$$
\boldsymbol{e}_{j}(a, b)=\boldsymbol{p}_{j, 1}+a \cdot\left(\boldsymbol{p}_{j, 2}-\boldsymbol{p}_{j, 1}\right)+b \cdot\left(\boldsymbol{p}_{j, 3}-\boldsymbol{p}_{j, 1}\right)
$$

Where $\boldsymbol{p}_{j, 1 \ldots 3}$ are the three points of which a plane $j$ is constructed. By definition, the intersection $\boldsymbol{E}_{j} \cap \boldsymbol{g}_{i}$ lies on the plane, it is hence described by two unique solutions $a_{j, i}$ and $b_{j, i}$ 


$$
\boldsymbol{g}\left(d_{i, j}\right)-\boldsymbol{p}_{j, 1}=a_{j, i} \cdot\left(\boldsymbol{p}_{j, 2}-\boldsymbol{p}_{j, 1}\right)+b_{j, i} \cdot\left(\boldsymbol{p}_{j, 3}-\boldsymbol{p}_{j, 1}\right)
$$

As illustrated in Sup. 1, $\boldsymbol{g}\left(d_{i, j}\right)$ lies outside of the triangle $j$ if

$$
a_{j, i}<0 \quad \vee \quad b_{i, j}<0 \quad \vee \quad a_{j, i}+b_{i, j}>1
$$

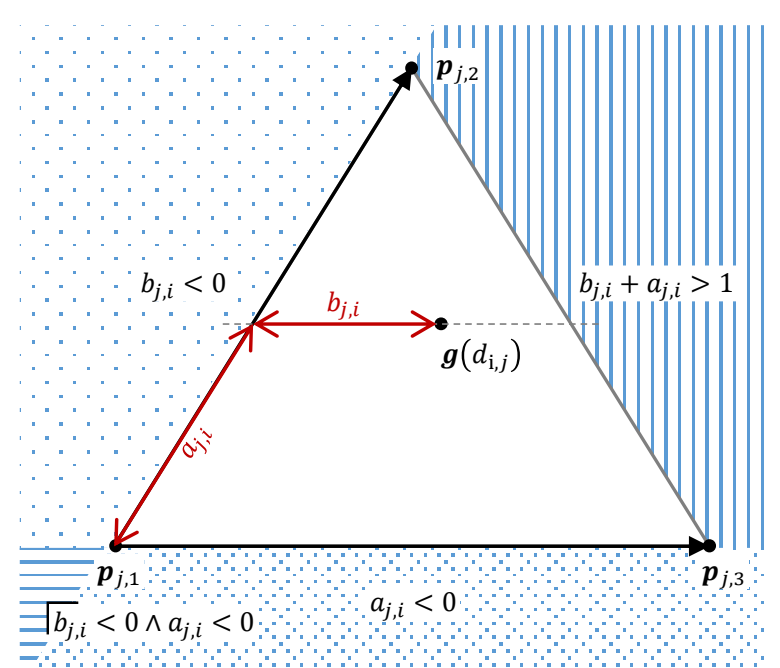

Sup. 1. Illustration of for which cases $\boldsymbol{g}\left(d_{i, j}\right)$ lies within or outside a triangle.

The problem was solved by calculating the dot product of equation (S5) with $\left(\boldsymbol{p}_{j, 2}-\boldsymbol{p}_{j, 1}\right)$ and $\left(\boldsymbol{p}_{j, 3}-\boldsymbol{p}_{j, 1}\right)$. This yielded two equations that were solved for $a_{j, i}$ and $b_{i, j}$.

Doing these calculations for all $10^{10}$ possible combinations of $i$ and $j$ was quite computational expensive. Therefore, the algorithm focused on $N_{\text {neighbors,it }}$ closest triangles $j$ for each triangle $i$. This was done for all triangles $i$ with an initial $N_{\text {neighbors }, 0}=40$. For all triangles $i$ for which no distance was found the calculation was repeated for $N_{\text {neighbors }, i t}=N_{\text {neighbors }, 0} * i t^{2}$. The closest neighbors are determined using the Euclidean distance of the center points of the triangles $i$ and $j$.

For two example meshes $\left(n_{s} \times n_{l} \approx 10^{10}\right)$ the algorithm resulted in a reduction to about $10^{6}$ distance calculations which took roughly 2 min on a standard desktop computer. Because $n_{s} \approx$ $10^{5}$ this indicates that only $n_{s} \times 10$ distance calculations were necessary using our algorithm.

To compare the results of the displacements of different crystals, that were differently oriented, the distance vectors were rotated such that they fit to the geometric crystal model shown in Figure 2 of the main text. The rotation matrix that performs this rotation was determined by the algorithm $^{2}$ that is used to determine a geometric crystal representation. The algorithm was applied during the overlaying procedure (section 2.5 of the main text). 
An example of a resulting dataset is show in Sup. 2. The white spot in the center was caused by the removed glued region.
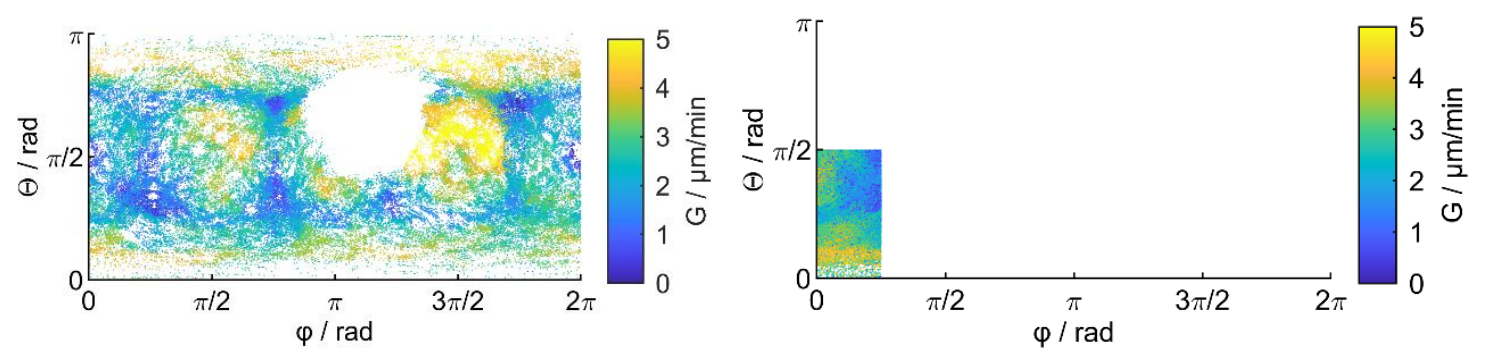

Sup. 2. Left: Average displacement rates of crystal 1 between 0 and $4 \mathrm{~min}$ in all growth directions. Right: The same dataset as shown in the left of this figure with applied symmetry conditions.

Note that PA has a cubic unit cell. ${ }^{3}$ Therefore, three symmetry operations were applied:

- Rotation by $\frac{\pi}{2}$ around the $\mathrm{z}$-axis ( $\varphi$-direction in spherical coordinates)

- Mirror symmetry with respect to a plane with the normal $\left(\begin{array}{lll}0 & 0 & 1\end{array}\right)^{T}\left(x y\right.$ plane, $\left.\theta=\frac{\pi}{2}\right)$

- Mirror symmetry with respect to a plane with the normal $\left(\begin{array}{llll}1 & 1 & 0\end{array}\right)^{T}\left(\varphi=\frac{\pi}{4}\right)$

The symmetry of PA is illustrated in Figure 2 of the main text. Note, however, that even though the unit cell is symmetrical, and, therefore, growth should occur symmetrically, this does not necessarily mean that the shape of crystals is also symmetrical. It was assumed that growth depends on the orientation of a normal with respect to the unit cell and, therefore, the symmetry conditions were applied by copying all measured displacements according to the symmetry conditions to the subspace defined by $0 \leq \varphi<\frac{\pi}{4}$ and $0 \leq \Theta<\frac{\pi}{2}$. Each time step yielded about $10^{5}$ data points. It is described in the main text that data of several time steps is used for fits using linear least squares. This yielded datasets of up to $10^{6}$ displacements. Such big datasets would use too much memory in the fitting procedure. This is why the displacement rates were summarized using the HEALPix algorithm. ${ }^{4}$ HEALPix devides a spherical surface in surface elements of equal surface area. The displacement rates were summarized by applying the mean to all data points within one of the surface elements defined by HEALPix. A grid resolution of 50 was used for HEALPix. This resulted in $12 \cdot 50^{2}=3 \cdot 10^{4}$ surface elements.

\section{Appendix II Choosing Order of Spherical Harmonics}

Eq. 1 in the main text defines the function that was chosen to describe the growth kinetics. The number of fit parameters $n$ in the equation is defined by the integer parameter $o \geq 0$ 


$$
n=o^{2}+2 o+1
$$

Functions with low $o$ will not be able to describe the datasets sufficiently. High $o$ on the other hand will lead to high calculation costs and may lead to overfitting. In order to choose an appropriate $o$, the growth rates of the first crystal between 0 and 4 min was fitted using varying $o \in\{0,10,20, \ldots, 70\}$. Sup. 3 shows the fit quality expressed as coefficient of determination $R^{2}$ together with the time needed to set up and solve the linear least squares problem over $o$. This illustrates that orders above 20 yield little improvement in respect to fit quality but come at a high computational cost. Therefore, we chose $o=20$ for this work.

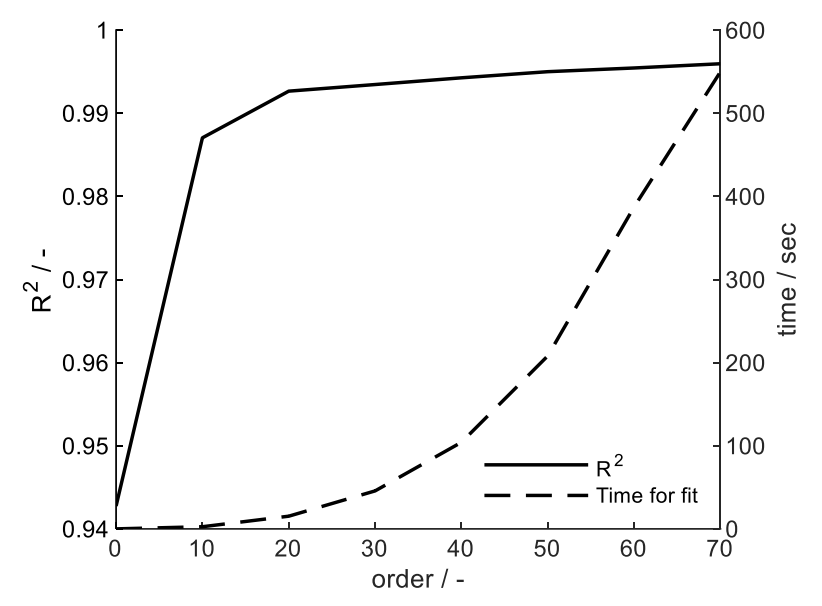

Sup. 3. Fit quality and time needed for setting up and solving the linear least squares problem.

\section{Appendix III Growth Rates over Time and Comparison with Literature Data}

In the following passage, we compare growth rates obtained from different crystals at different times. Because of the 3D nature of our datasets the visualization is not straightforward. We chose to compare growth in the classical face directions because these values can also be compared to data from the literature. Further, the fastest growth rates of the individual crystals are compared.

Sup. 4 shows the surface displacement rates of the crystals of the first experiment series for the $10 \%$ fastest rates $\left(G_{\max }\right)$, rates in [111] $\left(G_{111}\right)$, [110] $\left(G_{110}\right)$, and [100] $\left(G_{100}\right)$ directions, respectively. The slopes of the fitted lines (thick blue lines in Sup. 4) are quite low and result in changes over time that are similar to the standard deviations of the data points (Table 1). The figure, therefore, illustrates that - with the given measurement inaccuracies - these growth rates did not significantly vary over time. However, in some preliminary experiments we observed that especially the maximal growth rates might vary more at higher supersaturations. A more detailed study of other experimental conditions will be subject of our future work. The aim of this contribution is to establish our measurement method and growth model. 

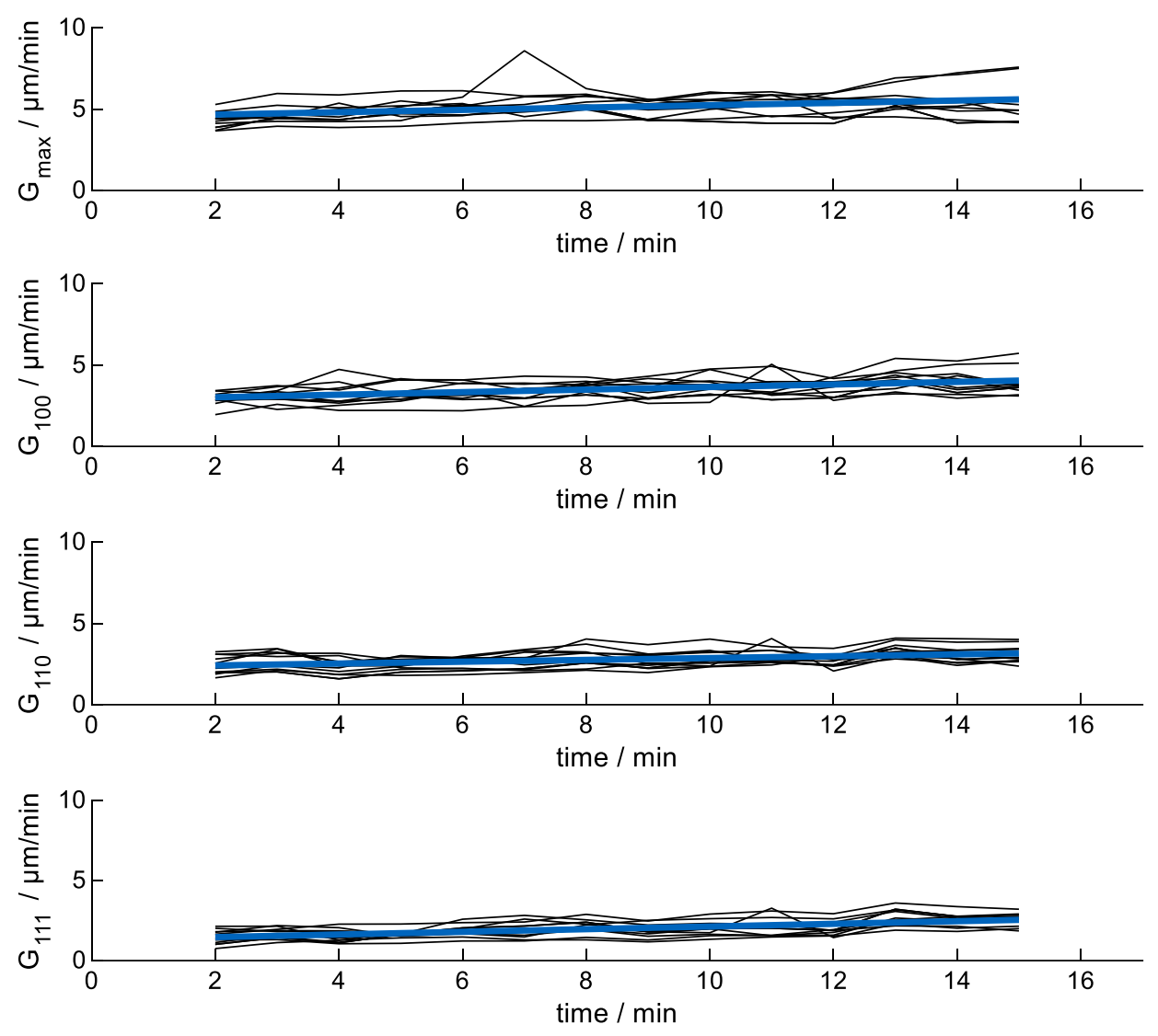

Sup. 4. Black lines: Mean surface displacement rates (moving average for a time interval of 4 min) over time for the $10 \%$ fastes rates, in [111], [110], and [100] directions, respectively. Error bars are not plotted for sakes of readability but are in the range of those plotted in Sup. 5; Thick blue lines: Fitted lines with slopes $0.07,0.08,0.06$ and $0.08 \mu \mathrm{m} / \mathrm{min}^{2}$, respectively.

For the scope of this contribution, the constant growth rates discard an alternative interpretation according to which growth could be governed by the crystals' size at a degree that would be captured by our method. This indicates that the surface displacement rates of the individual time steps can be combined. The mean face growth rates of all crystals of measurement series one are compared in Sup. 5. Sup. 5 shows that for most crystals the [111] faces grew with the slowest growth rates. There is one exception where [110] was the slowest. For all crystals, the [100] faces grew with the fastest growth rates. This is in agreement with the literature. ${ }^{3,5,6}$ Table 1 summarizes face specific growth rates from the literature. Even though the experiments in this study were conducted at the lowest supersaturation, the highest growth rates are measured. Here, the growth of abraded crystals was studied. Hence, the increased growth rates are in accordance with Ulrich et al. who reported increased growth in terms of mass for damaged 
crystals. ${ }^{7}$ A main working hypothesis of this work was that the abraded regions would grow at increased rates and faces would grow at their normal rates. However, the results indicate that the typically expressed faces grow at increased rates, too. A possible explanation might be increased strain and number of dislocations in the abraded crystals. ${ }^{6}$ However, variations of our measurements are high. The variation of the data from the literature is not reported. It is hence hard to judge the significance of the differences between our rates and those reported in the literature.

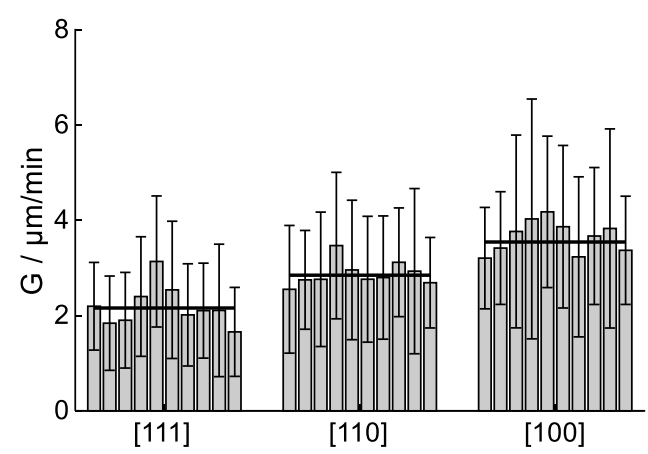

Sup. 5. Growth rates in face directions of individual crystals of experiment 1. Error bars indicate \pm 1 standard deviation $\left(n \sim 10^{5}\right)$. Horizontal lines indicate the mean values given in Table 1 .

Table 1. Face specific growth rates from this study and the literature. For the growth rates of this study, \pm 1 standard deviation $\left(n \sim 10^{6}\right)$ is given. Relative supersaturation is denoted by $\sigma$. Temperature is denoted by $\vartheta$. "Ma et al. ${ }^{3}$ studied a range of super saturations. Here, the lowest value (i.e. closest to our supersaturation) is given.

\begin{tabular}{|c|c|c|c|c|c|}
\hline & $\begin{array}{l}\sigma \\
-\end{array}$ & $\begin{array}{c}\vartheta \\
{ }^{\circ} \mathrm{C}\end{array}$ & $\begin{array}{c}G_{[111]} \\
\mu \mathrm{m} / \mathrm{min}\end{array}$ & $\begin{array}{c}G_{[110]} \\
\mu \mathrm{m} / \mathrm{min}\end{array}$ & $\begin{array}{c}G_{[100]} \\
\mu \mathrm{m} / \mathrm{min}\end{array}$ \\
\hline This study & 0.017 & 20 & $2.2 \pm 1.2$ & $2.8 \pm 1.3$ & $3.5 \pm 1.6$ \\
\hline Mullin et al. ${ }^{5}$ & 0.017 & 32 & 0.85 & - & $0.99 \ldots 1.85$ \\
\hline Matsuoka et al. ${ }^{8}$ & 0.022 & 25 & 0.62 & - & - \\
\hline Ma et al. ${ }^{3}$ & $0.05^{*}$ & $25 \ldots 40$ & 1.7 & 2.2 & 7.2 \\
\hline Ristic et al. ${ }^{6}$ & 0.064 & 34.55 & 1.2 & 2.5 & 1.9 \\
\hline
\end{tabular}




\section{Appendix IV Additional Figures}

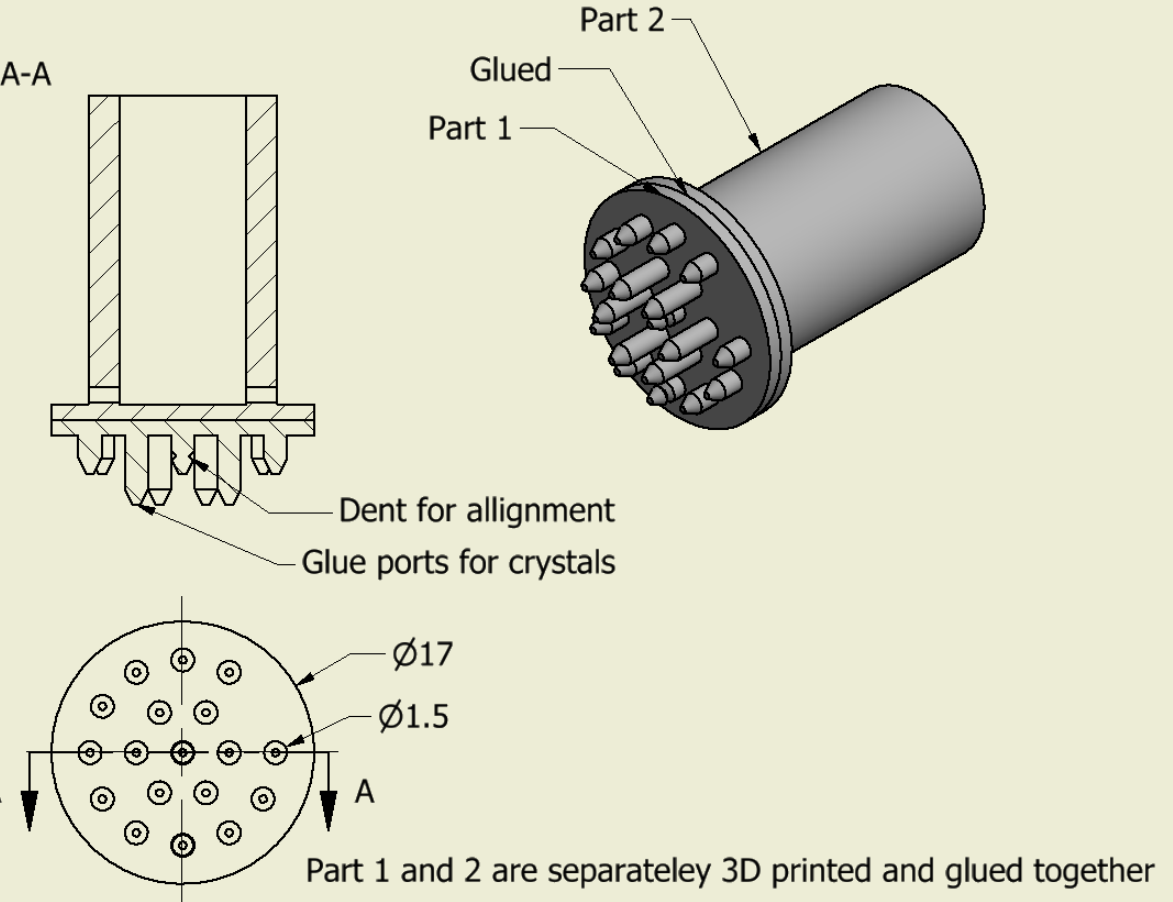

Sup. 6. Sketch of a growth rack: A rendered model of the growth racks is shown in the top right. A cut through the model (indicated by $\mathrm{A}-\mathrm{A}$ in the bottom) is shown in the top left. The top view is shown in the bottom of the image. The diameter of the rack is $17 \mathrm{~mm}$, the diameters of the pins are $1.5 \mathrm{~mm}$. All measures are given in $\mathrm{mm}$.

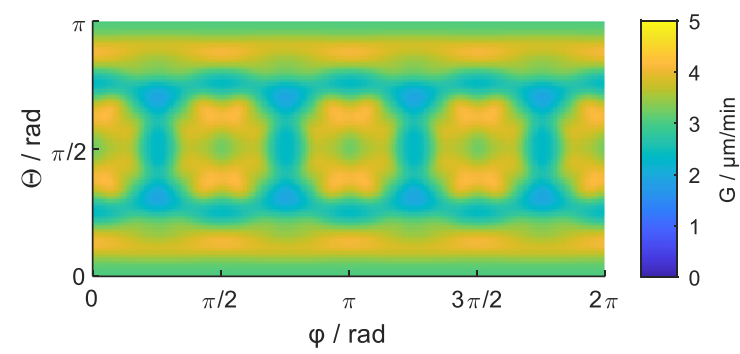

Sup. 7. Surface displacement rate of crystal 1.

\section{Appendix V References}

(1) Ericson, C. Real-time collision detection; Morgan Kaufmann series in interactive 3D technology; Elsevier Morgan Kaufmann, 2008.

(2) Kovačević, T.; Reinhold, A.; Briesen, H. Identifying Faceted Crystal Shape from ThreeDimensional Tomography Data. Cryst. Growth Des. 2014, 14 (4), 1666-1675. DOI: 10.1021/cg401780p. 
(3) Ma, C. Y.; Wan, J.; Wang, X. Z. Faceted growth rate estimation of potash alum crystals grown from solution in a hot-stage reactor. Powder Technol. 2012, 227, 96-103. DOI:

10.1016/j.powtec.2012.02.040.

(4) Gorski, K. M.; Hivon, E.; Banday, A. J.; Wandelt, B. D.; Hansen, F. K.; Reinecke, M.; Bartelmann, M. HEALPix: A Framework for High-Resolution Discretization and Fast Analysis of Data Distributed on the Sphere. Astrophys. J. 2005, 622 (2), 759-771. DOI: $10.1086 / 427976$.

(5) Mullin, J. W.; Garside, J. The crystallization of aluminium potassium sulphate: A study in the assessment of crystallizer design data Part I: Single crystal growth rates. Trans. Instn Chem. Engrs 1967, 45, 285-290.

(6) Ristic, R. I.; Shekunov, B.; Shewood, J. N. Long and short period growth rate variations in potash alum crystals. J. Cryst. Growth 1996, 160 (3-4), 330-336. DOI: 10.1016/00220248(95)00741-5.

(7) Ulrich, J.; Stepanski, M. Einfluß der Oberflächen-Beschaffenheit auf das Kristallwachstum aus Lösungen. Chem. Ing. Tech. 1987, 59 (5), 402-404. DOI: 10.1002/cite.330590508.

(8) Matsuoka, M.; Abe, Y.; Uchida, H.; Takiyama, H. Mechanism of growth rate enhancement by micro-crystals for the potash alum-water system. Chem. Eng. Sci. 2001, 56 (7), 2325-2334. DOI: 10.1016/S0009-2509(00)00453-X. 